\title{
Real-time magnetic resonance imaging-guided transcatheter aortic valve replacement
}

\author{
Justin G. Miller, MD, Ming Li, PhD, Dumitru Mazilu, PhD, Tim Hunt, BS, and Keith A. Horvath, MD
}

\section{ABSTRACT}

Objectives: To demonstrate the feasibility of Real-time magnetic resonance imaging (rtMRI) guided transcatheter aortic valve replacement (TAVR) with an active guidewire and an MRI compatible valve delivery catheter system in a swine model.

Methods: The CoreValve system was minimally modified to be MRI-compatible by replacing the stainless steel components with fluoroplastic resin and highdensity polyethylene components. Eight swine weighing 60-90 kg underwent rtMRI-guided TAVR with an active guidewire through a left subclavian approach.

Results: Two imaging planes (long-axis view and short-axis view) were used simultaneously for real-time imaging during implantation. Successful deployment was performed without rapid ventricular pacing or cardiopulmonary bypass. Postdeployment images were acquired to evaluate the final valve position in addition to valvular and cardiac function.

Conclusions: Our results show that the CoreValve can be easily and effectively deployed through a left subclavian approach using rtMRI guidance, a minimally modified valve delivery catheter system, and an active guidewire. This method allows superior visualization before deployment, thereby allowing placement of the valve with pinpoint accuracy. rtMRI has the added benefit of the ability to perform immediate postprocedural functional assessment, while eliminating the morbidity associated with radiation exposure, rapid ventricular pacing, contrast media renal toxicity, and a more invasive procedure. Use of a commercially available device brings this rtMRI-guided approach closer to clinical reality. (J Thorac Cardiovasc Surg 2016;151:1269-77)

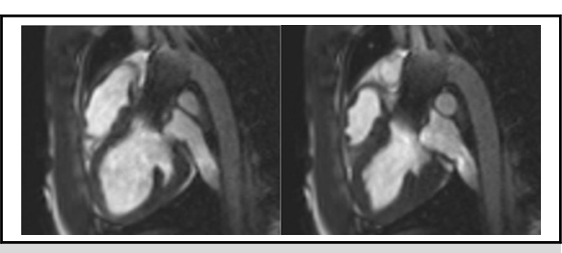

Postdeployment cine MRI to confirm valve position Left, diastole; right, systole.

\section{Central Message}

We demonstrate the feasibility of rtMRI-guided TAVR, which has benefits over current imaging technologies.

\section{Perspective}

Real-time MRI-guided TAVR overcomes the limitations of the current imaging modalities. This method provides superior visualization and deployment with pinpoint accuracy while eliminating the morbidity of radiation exposure, rapid ventricular pacing, and contrast media renal toxicity.

See Editorial Commentary page 1278.
Aortic stenosis is the most common type of valvular heart disease in the United States. ${ }^{1-4}$ While the disease process has a long latency period, patients rapidly decline after they become symptomatic unless they undergo valve replacement. $^{5-7}$ Unfortunately, some of these patients are not suitable surgical candidates or are at high risk. ${ }^{8}$ Without valve replacement, the mortality for these patients after onset of symptoms approaches 50\% after 2 years and $80 \%$ after 5 years. 9,10 The advent of transcatheter aortic valve replacement (TAVR) has become a viable treatment option for patients with aortic stenosis patients who are considered otherwise inoperable or at

From the Cardiothoracic Surgery Research Program, National Heart, Lung and Blood Institute, National Institutes of Health, Bethesda, Md.

This research was funded by the National Heart, Lung and Blood Institute, Division of Intramural Research.

Read at the 41st Annual Meeting of The Western Thoracic Surgical Association, Whistler, British Columbia, Canada, June 24-27, 2015.

Received for publication July 23, 2015; revisions received Oct 8, 2015; accepted for publication Nov 15, 2015; available ahead of print Dec 24, 2015

Address for reprints: Keith A. Horvath, MD, 10 Center Dr, Bldg 10, Rm B1D47, Bethesda, MD 20892 (E-mail: horvathka@mail.nih.gov).

$0022-5223 / \$ 36.00$

Copyright (C) 2016 by The American Association for Thoracic Surgery

http://dx.doi.org/10.1016/j.jtcvs.2015.11.024 high risk. ${ }^{8,11}$ Two devices are currently available for use in the United States: the CoreValve System (Medtronic, Minneapolis, Minn) and the SAPIEN Transcatheter Heart Valve (Edwards Lifesciences, Irvine, Calif). ${ }^{12-14}$

Although there have been multiple advances in valve design and valve delivery technology, imaging modalities have remained largely unchanged. TAVR is currently an intricate procedure that requires multimodality imaging including preprocedural imaging for planning, intraprocedural imaging for guidance, and postprocedural imaging for confirmation of placement. $^{15,16}$ Currently, the preprocedural evaluation usually includes echocardiography in combination with multidetector computed tomography (CT) or CT angiography. The TAVR procedure is most commonly performed using a combination of fluoroscopy and transesophageal echocardiography (TEE). Postprocedural

Scanning this QR code will take you to the article title page.

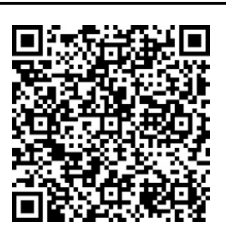




\section{Abbreviations and Acronyms \\ CT = Computed tomography \\ Gd-DTPA $=$ Gadolinium with diethylenetriamine penta-acetic acid \\ $\mathrm{RF} \quad=$ Radiofrequency \\ rtMRI = Real-time magnetic resonance imaging \\ TAVR $=$ Transcatheter aortic valve replacement \\ TEE $=$ Transesophageal echocardiography}

imaging also routinely uses a combination of fluoroscopy and TEE to confirm valve placement and cardiac function. ${ }^{15,17-19}$ Fluoroscopy has multiple limitations, including poor soft tissue contrast, a requirement for rapid ventricular pacing, radiation exposure to the patient and surgical team, and contrast-induced nephropathy. ${ }^{14,20-22}$ Real-time magnetic resonance imaging (rtMRI) guidance overcomes these limitations with improved 3-dimensional visualization of the anatomic structures, guidewires, delivery catheter system, and bioprosthetic valve while allowing delivery of the valve with pinpoint accuracy. In addition, the use of rtMRI allows for immediate postprocedural functional assessment. ${ }^{22-24}$

Our group has successfully performed TAVR procedures through a transapical approach under rtMRI guidance. We have proven that this is a reproducible method with a high accuracy of device delivery. ${ }^{14,22,25}$

In the present study, we have demonstrated that the CoreValve system can be easily and effectively deployed with a left subclavian approach using rtMRI guidance, a minimally modified valve delivery catheter system, and an active guidewire. We report results indicating preclinical feasibility in an acute, nonsurvival swine model. To date, no device clinically available in the United States has been used for rtMRI TAVR.

\section{METHODS}

\section{Medtronic CoreValve System}

The Medtronic CoreValve is an aortic bioprosthetic transcatheter heart valve consisting of 3 porcine pericardial leaflets sutured to a self-expanding, multilevel, radiopaque nitinol frame (Figure 1). ${ }^{21,26}$ The porcine pericardial leaflets are processed with alpha-amino oleic acid, an antimineralization treatment derived from a naturally occurring long-chain fatty acid. ${ }^{26}$ The nitinol stent is manufactured by laser cutting a nitinol tube. ${ }^{27}$ The stent is designed with 3 sections. The lower section has a high radial force, to anchor the valve and displace the calcified leaflets. The middle section holds the 3 porcine pericardial leaflets and is of smaller diameter than the lower and upper sections, to avoid occluding the coronary arteries, which largely eliminates the need to adjust the rotational position on deployment. The upper section has the largest diameter and exerts a lower radial force. The design of this section allows the stent to self-center during placement. ${ }^{21,27}$ The commercially available version of the CoreValve is available in several different sizes $(23,26,29$, and $31 \mathrm{~mm}$ ), which correlates with annulus diameters from 18 to $29 \mathrm{~mm}$ and ascending aorta diameters up to $43 \mathrm{~mm}$. The device is MRI conditional, and nonclinical testing to date has confirmed that it is safe to use in MRI. ${ }^{21,26}$

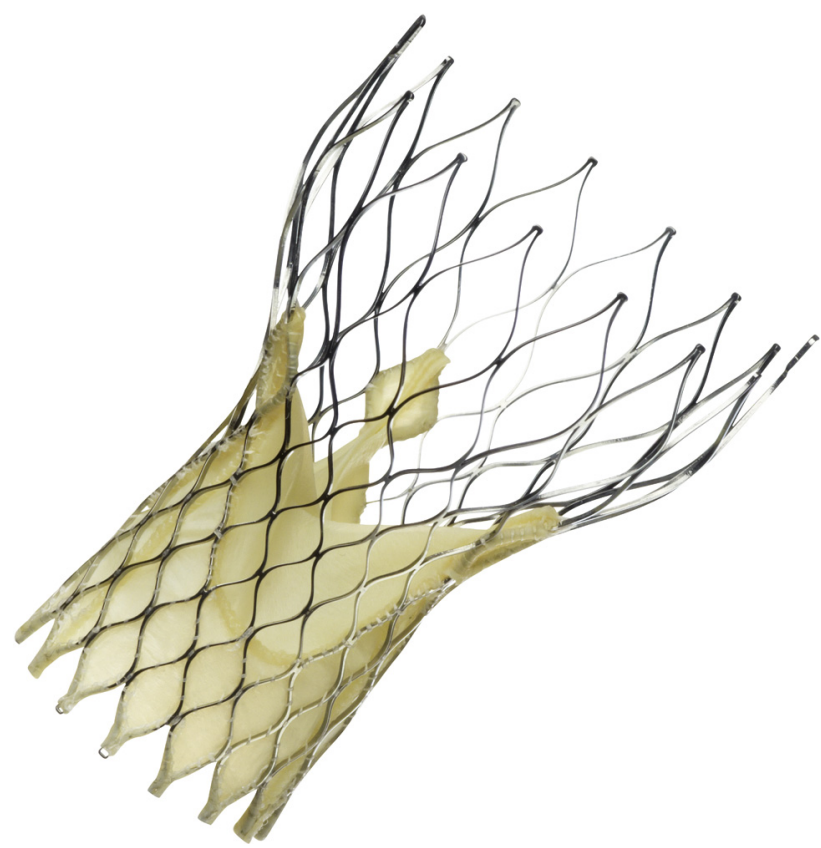

FIGURE 1. Medtronic CoreValve.

\section{Medtronic CoreValve Delivery Catheter System}

The CoreValve delivery catheter system is compatible with a $0.035^{\prime \prime}$ guidewire. The distal end of the delivery catheter system has an atraumatic, radiopaque tip and a capsule that covers and maintains the valve in a crimped position. The handle is located on the proximal end of the delivery catheter system and is used to load and deploy the valve. The handle consists of a slider to open and close the capsule, with a knob to facilitate slow opening of the slider to allow precise placement. The device also has an outer tube, the AccuTrak stability layer. This outer tube covers the catheter sheath to protect the retractable delivery catheter system, introducer sheath, and vessel walls, and allows the catheter to retract freely and provides a more stable platform for deployment. The first generation model of this delivery catheter system was a $24 \mathrm{Fr}$ sheath; however, the device is currently in its third generation and is $18 \mathrm{Fr}$. Now the outer diameter of the catheter is $15 \mathrm{Fr}$ and the outer diameter of the valve capsule is $18 \mathrm{Fr}$. The delivery catheter system is approved for femoral, subclavian, axillary, and ascending aortic access sites, and is not MRI-compatible. ${ }^{21,26}$

\section{Delivery Catheter System Modification}

The Medtronic CoreValve delivery catheter system was modified to be MRI-compatible. The delivery catheter system sheath has an inner and outer flexible polymer tube embedded with braided stainless steel. The sheath also contains an inner shaft that has metallic components. To make the delivery catheter system MRI-compatible, the inner tube and capsule were replaced with tubes made of fluoroplastic resin. The outer tube was replaced with a tube from a 14 Fr Check-Flo Performer Introducer (Cook Medical, Bloomington, Ind). The metallic components in the delivery catheter system handle were replaced with corresponding parts composed of plastic materials. In addition, the handle was redesigned with a thumb slider to open and close the capsule instead of the original design composed of a slider and knob.

The delivery catheter system modifications were implemented to maintain device performance, including rigidity and flexibility, while achieving MRI compatibility. With the replacement of the non-MRIcompatible components with MRI-compatible components, the outer diameter of the catheter was increased from $15 \mathrm{Fr}$ to $18 \mathrm{Fr}$ and the outer 

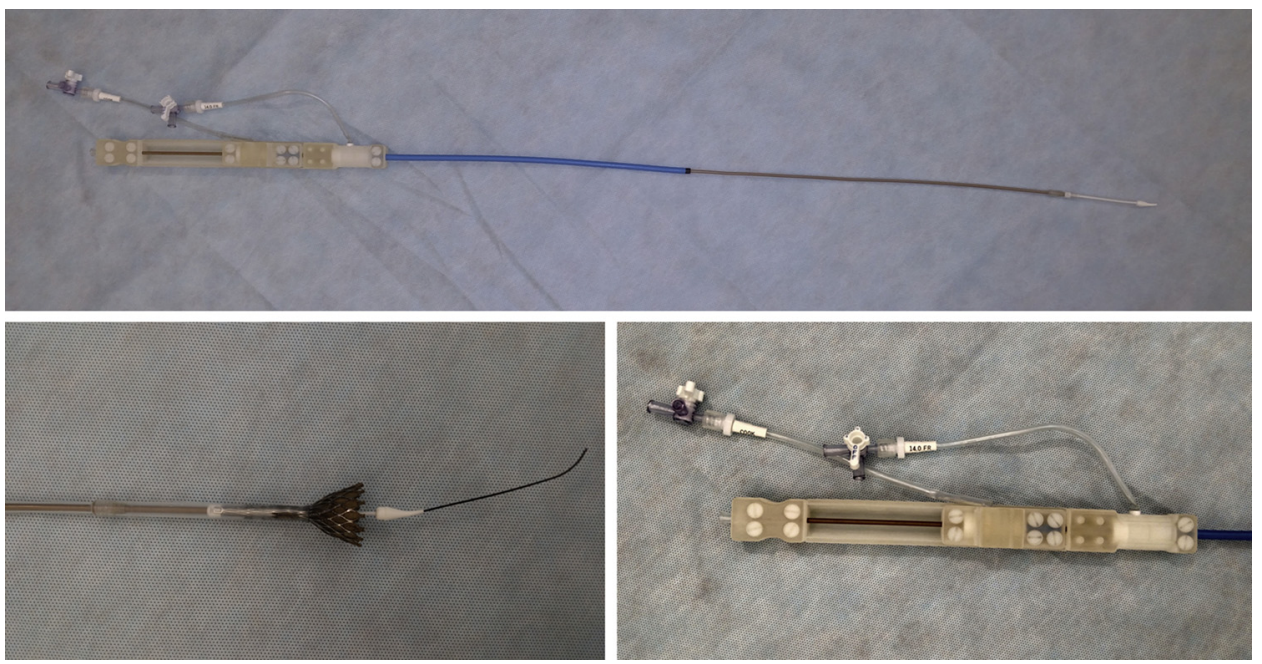

FIGURE 2. Top and bottom right, Modified delivery catheter system. Bottom left, Valve deployment with active guide wire.

diameter of the valve capsule was increased from $18 \mathrm{Fr}$ to $20 \mathrm{Fr}$. The modified delivery catheter system fit into a $20 \mathrm{Fr}$ introducer (Figures 2 and 3).

\section{Active Guidewire}

An active guidewire was developed to guide the delivery catheter system before deployment. The active guidewire included an MRI loop coil antenna, manufactured using insulated $0.005^{\prime \prime}$ magnet copper wire (Heraeus Medical Components, St Paul, Minn) in an ISO class 7 cleanroom. The coil length was adjusted to $1.1^{\prime \prime}$ with a $0.026^{\prime \prime}$ outer diameter. A $0.006^{\prime \prime}$ profile twisted pair served as a transmission line for the loop coil antenna. The whole structure was insulated using medical-grade polyester heat shrink tubing. The loop coil antenna was matched to $50 \Omega$ and tuned to the Lamoure frequency of a 1.5-T MRI scanner. The matching and decoupling circuit box was attached to the loop coil antenna by MMCX type radiofrequency (RF) connectors (Microstock, West Point, Pa) (Figure 3). ${ }^{13,28}$

\section{Imaging Technology}

All procedures and imaging were performed in a hybrid operating room suite using a 1.5-T Magnetom Aera imaging system (Siemens Medical Solutions, Munich, Germany). The magnet design includes a short 145 -cm-long by $70-\mathrm{cm}$-wide bore, which is large enough to provide surgical access to the animal within the magnet. Imaging system components include an interactive user interface, a large-screen display, advanced pulse sequence technology, and image reconstruction software. $^{29-31}$

Interactive Front End navigation software (Siemens Corporate Research, Munich, Germany), along with an interactive real-time pulse sequence (BEAT_IRTTT), provided real-time navigation for valve deployment. The system not only displays standard MRI images, but also has a fully interactive, real-time imaging system. The image reconstruction software obtains multiple slices in rapid succession that can be displayed simultaneously to provide a 3-dimensional rendering. These imaging planes can be quickly adjusted, allowing for real-time interpretation of anatomic structures and device tracking. ${ }^{29-33}$

\section{Animal Surgery Protocol}

All experiments were performed in accordance with protocols approved by the National Institutes of Health's Animal Care and Use Committee. Induction included an intramuscular injection of midazolam
$(0.5 \mathrm{mg} / \mathrm{kg})$ and ketamine $(25 \mathrm{mg} / \mathrm{kg})$. After induction, the animals were intubated and then maintained on mechanical ventilation with isoflurane $(0.5 \%-2.5 \%)$. End-tidal carbon dioxide, oxygen saturation, arterial blood pressure, and electrocardiographic telemetry were monitored throughout the entire procedure. Body temperature was maintained with a forced-air warming blanket.

Before the start of the procedure, the animals received intravenous amiodarone (150-300 mg) for antiarrhythmic treatment, and before trocar insertion they received anticoagulation therapy with heparin $(300 \mathrm{U} / \mathrm{kg})$. After completion of the experiment, the fully anesthetized animals were euthanized with an intravenous injection of phenobarbital $(150 \mathrm{mg} / \mathrm{kg})$. The times and details of all procedures were recorded.

\section{Valve Deployment}

Eight swine each weighing between 60 and $90 \mathrm{~kg}$ underwent rtMRI-guided TAVR with the Medtronic CoreValve. The animals were sedated, intubated, prepped and draped in a supine position, as described previously. A 4-cm left subclavian cutdown was performed. Vessel loops were placed around the subclavian for proximal and distal vascular control. A purse string suture was placed in the subclavian for closure postprocedure. An incision was made in the subclavian artery, into which the $20 \mathrm{Fr}$ sheath was inserted. The swine were then transferred to the MRI machine, and a surface RF coil was secured to the anterior chest to enhance signal reception. A predeployment scan was performed to confirm annulus and aortic root size

The MRI-compatible delivery catheter system was then loaded with the appropriately sized CoreValve. Two imaging planes (long-axis and short-axis views) were used to create a virtual real-time 3D reconstruction, which the surgeon could view from a projector screen in the MRI suite. The delivery catheter system was loaded over the guidewire, which was then introduced into the sheath and advanced until it was across the aortic valve (Figure 4, A). The delivery catheter system was then advanced under rtMRI guidance (Figure 4, B). Once the device reached the proper position, the capsule was slowly retracted (Figure 4 C, D, and E). Before deployment, repositioning of the bioprosthesis was performed if necessary to ensure correct and precise placement. Once the capsule was fully retracted, the valve was deployed and the delivery catheter system was withdrawn from the sheath (Figure 4, F). Throughout the procedure, the surgeon was in direct communication with the scanner operator via headphones and a microphone (Magnacoustics, Atlantic Beach, NY) to request any changes in the imaging planes as needed to visualize the device and ensure proper placement. $^{13,22,31}$ 

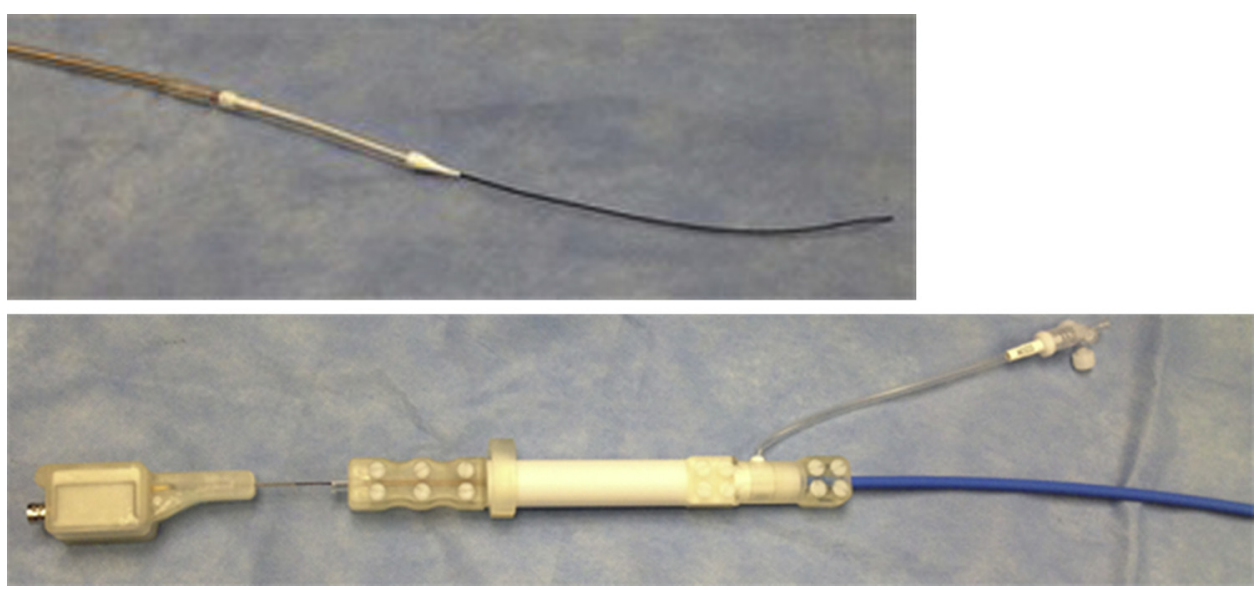

FIGURE 3. Top, The delivery system and active guide wire. Bottom, The tip of the delivery system and the active guide wire, showing the handle of the delivery system and the RF connector.

\section{Postdeployment Valve Assessment}

After removal of the delivery catheter system, postdeployment images were acquired to confirm the position of the prosthesis and to evaluate valvular and global heart function. Gated cine MRI was used to assess aortic valve function and left ventricular function (Figure 5), and phase-contrast cine MRI was used to assess blood flow through the newly implanted bioprosthesis and to evaluate for intravalvular or paravalvular regurgitation. A magnetic resonance first-pass perfusion scan was also performed in several animals, with an intravenous injection of gadolinium with diethylenetriamine penta-acetic acid (Gd-DTPA; Magnavist, Berlex, Montville, NJ) contrast medium to assess coronary blood flow (Figure 6). Necropsy was performed on all animals after valve placement, along with a postdeployment functional scan to grossly assess valve placement (Figure 7). ${ }^{22,29,31,34}$

\section{RESULTS \\ Valve Deployment}

All 8 swine underwent successful MRI-guided TAVR with the Medtronic CoreValve. The mean time taken for the left subclavian cutdown with sheath placement was $21 \pm 9$ minutes. The predeployment scan was used to measure the annulus and aortic root size to determine valve selection. The sequences were measured in an oblique sagittal view, which corresponded to a similar view seen with a parasternal long-axis view via transthoracic echocardiogram and a midesophageal long-axis view via TEE for valve measurement. ${ }^{35,36}$ The annulus and aortic root sizes were $26 \pm 2 \mathrm{~mm}$ and $22 \pm 3 \mathrm{~mm}$, respectively. A 26- $\mathrm{mm}$ CoreValve bioprosthesis was used for all 8 experiments. An active guidewire was used in 6 experiments, and a passive Nitrex guidewire (EV3, Plymouth, Minn) was used for the other 2 experiments. This passive guidewire was an 0.035 -in, $180-\mathrm{cm}$ angled hydrophilic guidewire. There were no issues with valve deployment. The mean time to place the guidewire was $60 \pm 10$ seconds, and the mean time to deploy the valve under rtMRI guidance was $25 \pm 3$ seconds.

\section{Postdeployment Valve Assessment}

The postdeployment scans confirmed precise valve placement in all 8 experiments, and also confirmed global and regional cardiac function (Figure 5). The timeresolved velocity of blood flow through the valve was measured using a cine phase-contrast MRI sequence in short-access planes proximal and distal to the valve. These imaging sequences confirmed adequate systolic blood flow with optimal valve functioning. There was no evidence of turbulent blood flow, regurgitation, or paravalvular leak. A magnetic resonance first-pass perfusion scan was performed in 2 animals with intravenous injection of Gd-DTPA, which confirmed adequate coronary perfusion (Figure 6). The necropsy results confirmed the MRI findings (Figure 7). On necropsy, all eight animals had apposition of the bioprosthesis to the annulus and ascending aorta. All valves were deployed in the correct position, with the proximal end 4-6 mm below the annulus and not occluding the coronary ostia (Figure 7).

\section{DISCUSSION}

Our group and others have demonstrated that rtMRI can serve as the sole imaging modality for predeployment imaging, imaging guidance for valve deployment, and postdeployment scanning in TAVR procedures with reproducible and highly accurate device delivery. ${ }^{13,22,25,30,31,37,38}$ rtMRI-guided TAVR has come closer to a clinical reality over the past decade. We have modified our methodology with the knowledge gained from multiple experiments to create what we believe is a preclinical model that can be translated to a clinical trial.

Our first experiment used a commercially available stentless valve mounted on a custom-designed, balloonexpandable platinum stent. The valves were implanted via a transapical approach in 8 swine with a custom-designed delivery device. The results confirmed the feasibility 


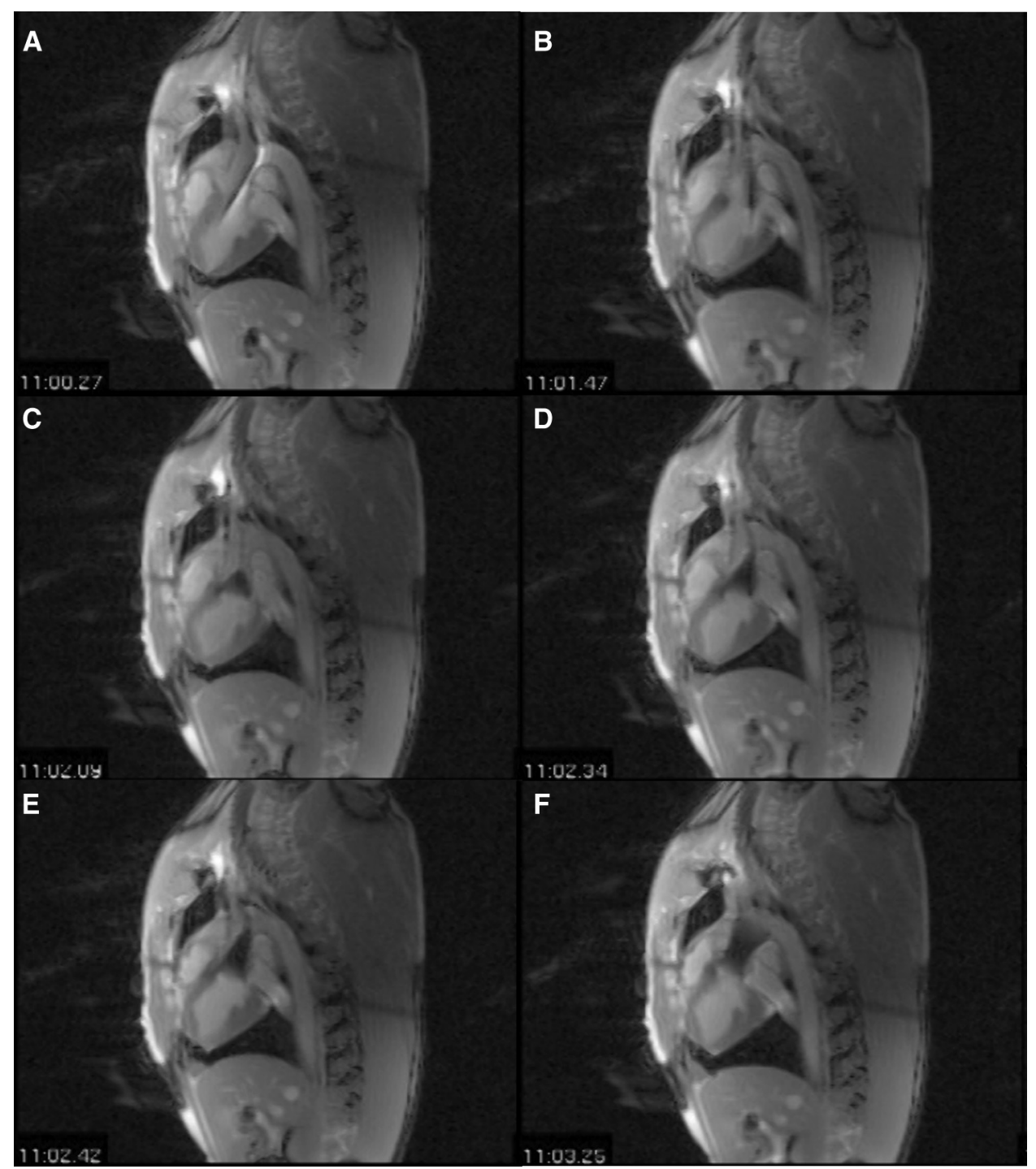

FIGURE 4. Long-axis view of rtMRI-guided valve deployment. A, Guide wire advanced across aortic valve. B, Delivery catheter system advanced over guide wire and into position. C, D, and E, Retracting capsule to deploy valve. F, Valve deployed.

of transapical TAVR with rtMRI guidance. ${ }^{30}$ The next experiment in our series again used a commercially available stentless valve mounted on a custom-designed, balloon-expandable platinum stent. The valves were also implanted via an apical approach in 28 swine with a custom-designed delivery device. Ten of the swine were allowed to survive to 1,3 , and 6 months with intermittent imaging. Long-term results demonstrated the stability of the implants. ${ }^{22}$ We also compared balloon-expandable stents and self-expandable stents mounted in commercially available valves. These valves were implanted via a transapical approach in 22 swine. The swine were allowed to survive to 1,3 , and 6 months with intermittent imaging. We concluded that self-expanding stents were easier to deploy under rtMRI guidance and had fewer complications. ${ }^{25}$ As the clinically approved technology developed, our group then performed an experiment using a commercially available balloon-expandable valve and minimally modified valve delivery device. We implanted the valve in 8 swine via a transapical approach. The results of this experiment confirmed the feasibility of deploying a commercially available balloon-expandable stent with a minimally modified delivery device via a transapical approach. ${ }^{13}$

Although the foregoing experiments demonstrated the feasibility of an apical approach, we wanted a commercially available, self-expanding device that would be amenable to delivery via a transcatheter approach. We then performed a feasibility experiment with our active guidewire for transapical deployment of a self-expandable stents mounted in commercially available valves in 16 swine. Six of the swine were allowed to survive to 90 days. The results of this 

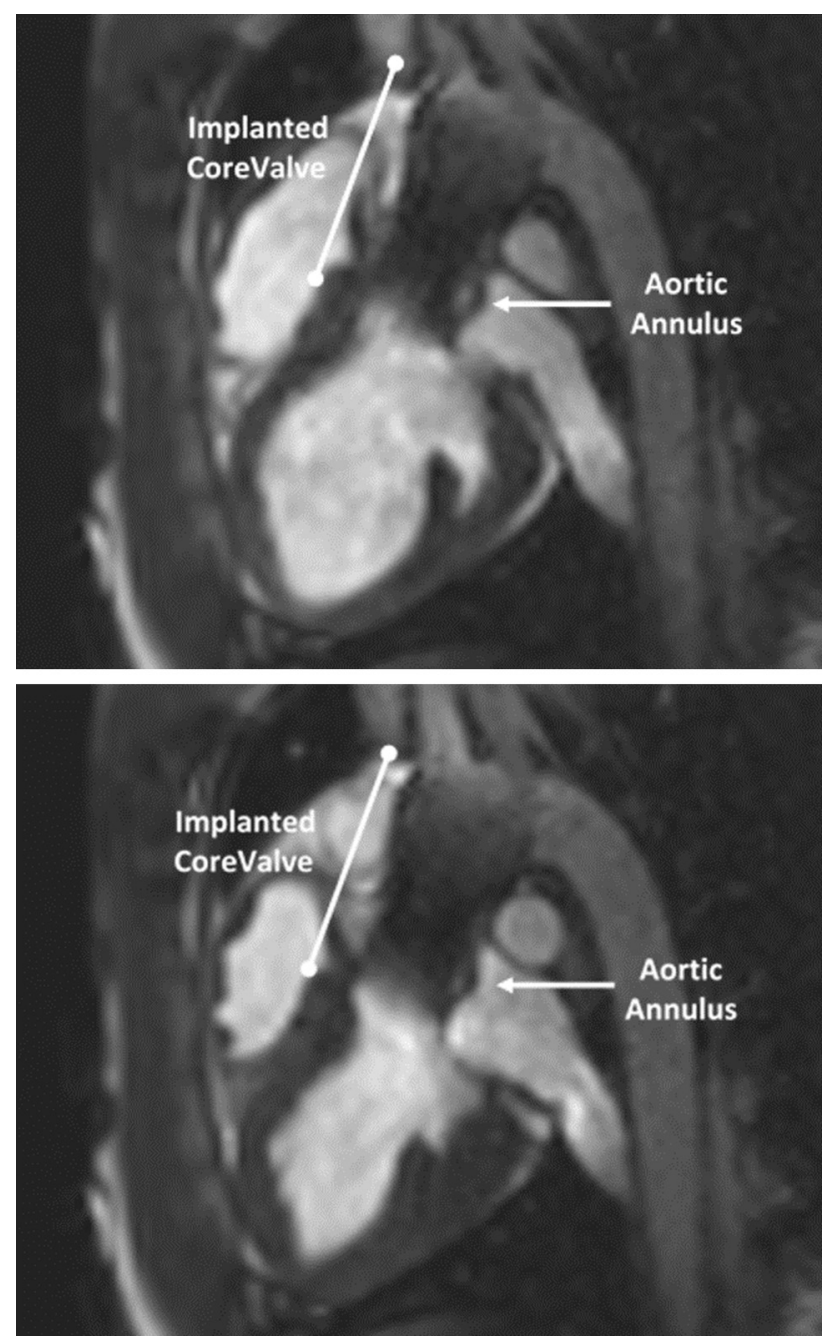

FIGURE 5. Postdeployment CINE (long-axis view) to confirm valve position. Top, diastole; bottom, systole.

experiment confirmed the benefit of an active guidewire, as well as the stability of our custom-designed stent over time. ${ }^{14}$ This led us to our current experiment, which demonstrates the feasibility of using rtMRI guidance with a minimally modified valve delivery catheter system, a commercially available bioprosthetic valve, and an active guidewire for TAVR.

Whereas the CoreValve nitinol stent is MRI-compatible, the Medtronic delivery device required some modification to be MRI-compatible. Our delivery catheter system modifications were carefully implemented to maintain device performance, including rigidity and flexibility, while achieving MRI compatibility. As part of the design modifications, the substituted inner tube and capsule were slightly thicker than the original components, because the fluoroplastic resin that we used did not have braided stainless steel to provide rigidity. As a result, the outer diameter of the valve capsule was increased from fitting in an $18 \mathrm{Fr}$ introducer sheath to a $20 \mathrm{Fr}$ introducer sheath. The increased sheath size was not a limitation for our surgical subclavian cutdown. Because our experiment used an acute nonsurvival model, surgical repair of the subclavian was not performed. For a transition to a clinical model, we do not anticipate the increased profile to be a limitation, given the existence of percutaneous closure devices, such as the Perclose ProGlide device, that are approved to close $20 \mathrm{Fr}$ access sites.

We also found that an active guidewire significantly improved its visualization while advancing across the aortic valve, and also reduced the time required to advance the guidewire. The thin profile of a guidewire makes it difficult to visualize on MRI. The active guidewire overcomes this limitation by being visualizable even when moved outside the imaging slice. ${ }^{33,39}$ An active guidewire with a loop coil antenna was used. The guidewire was then assigned a unique image signature, allowing the user to choose a color with the imaging software to match the image signature. We chose neon green because it was readily visible when imposed on the darker rtMRI images. This greatly aided visualization of guidewire advancement across the aortic valve. During one of our experiments, the connector between the guidewire and the RF connector was damaged, so we used a passive nitrex guidewire (EV3) for 2 experiments. The time required to advance the passive guidewire across the aortic valve was approximately 2 minutes in both experiments, whereas the average time to advance the passive and active guidewires in all 8 experiments was $60 \pm 10$ seconds. It took extra time to adjust the long-axis imaging plane to visualize the tip of the catheter in the imaging slice. As the passive guidewire was advanced, the long-axis plan had to be continually readjusted. In addition, visualization of the passive guidewire was limited, and artifact obscured the 2 imaging planes. As seen in our experiments, passive guidewires have multiple limitations, including poor contrast and excessive artifacts. ${ }^{29}$ Once the delivery device was advanced over the guidewire, the active guidewire again aided visualization of the delivery device.

Although CT is the most accepted imaging modality for preprocedural imaging, MRI provides comparable information about cardiac and aortic anatomy and geometry. ${ }^{40-42}$ In our experiments, the MRI predeployment scan was helpful in confirming annulus and aortic root sizes before starting the procedure. Because of the superior soft tissue contrast and improved visualization, rtMRI has the added benefit of allowed prompt recognition of periprocedural complications such as dissection, perforation in the aorta or left ventricle from the tip of the delivery device, or dislocation of the prosthetic valve into the ascending aorta. Our experiment also included a postdeployment scan not only to confirm valve placement, but also to confirm global and regional cardiac function. We used a cine 


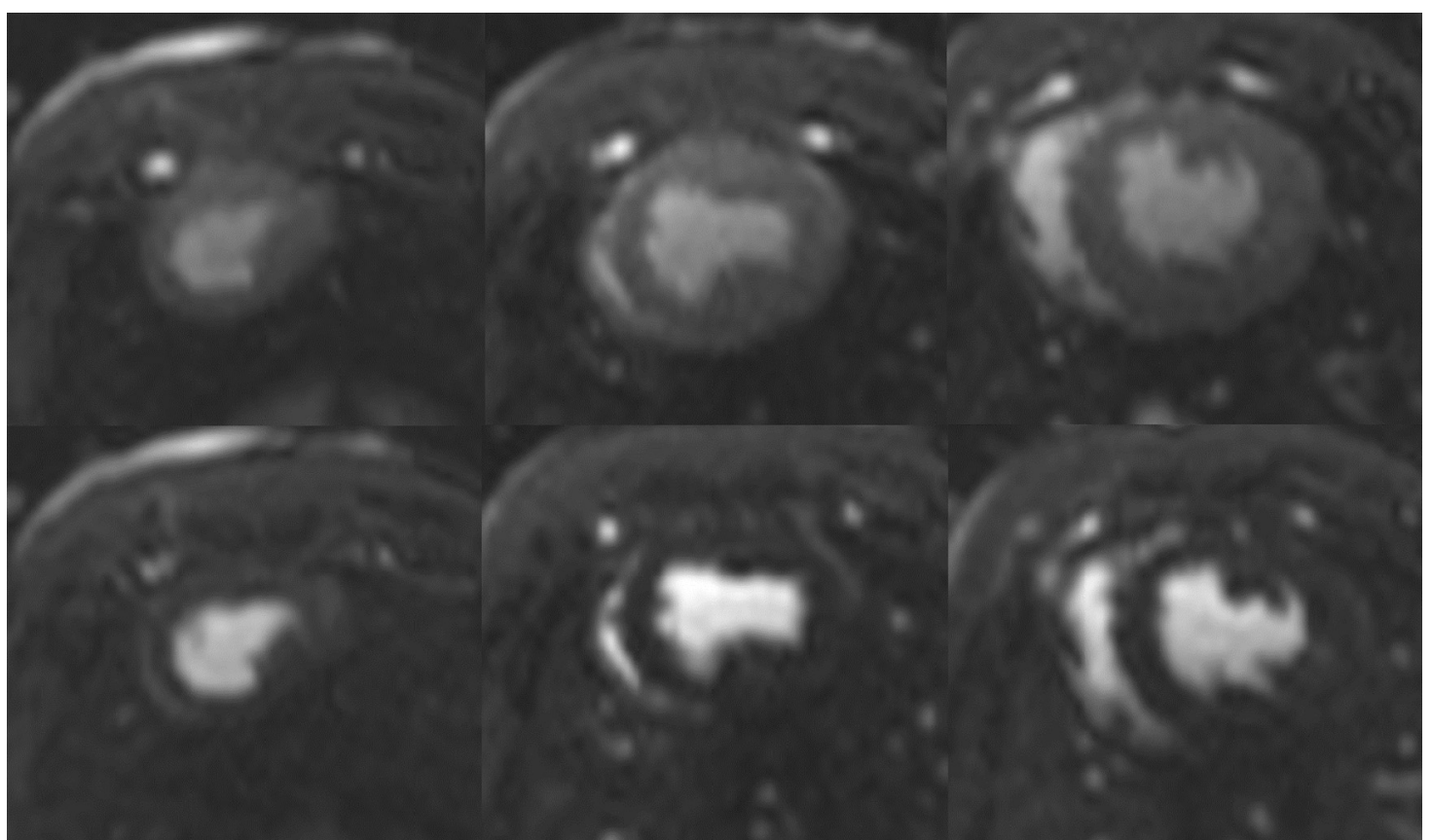

FIGURE 6. Postdeployment perfusion scan. Left column, apex; middle column, mid-wall; right column, base. Top row, arterial phase; bottom row, venous phase.

phase-contrast MRI sequence to measure the time-resolved velocity of blood flow through the valve. All 8 of our experiments had adequate systolic blood flow with optimal valve functioning. There was no evidence of turbulent blood flow, regurgitation, or paravalvular leak. We also performed a magnetic resonance first-pass perfusion scan in 2 animals with intravenous injection of Gd-DTPA, which confirmed adequate coronary perfusion. We also performed necropsy on all animals, because this was an acute, nonsurvival model. Necropsy was able to confirm the MRI findings with apposition of the bioprosthesis to the annulus and ascending aorta. In addition, all valves were deployed precisely, with the proximal end 4-6 $\mathrm{mm}$ below the annulus and without occluding the coronary ostia.
rtMRI has multiple benefits over the current imaging modality of echocardiography and fluoroscopy. X-ray fluoroscopy exposes the patients and medical staff to significant doses of ionizing radiation. Conversely, rtMRI uses nonionizing radiation with no known genetic effects or changes in chemical binding and no known deleterious effects to health. ${ }^{43}$ rtMRI-guided TAVR using the CoreValve does not require the use of rapid ventricular pacing to aid ventricular unloading, because the valve design does not occlude transaortic blood flow during deployment. Although balloon aortic predilation with rapid ventricular pacing is used before CoreValve deployment, several authors have found similar outcomes without predilation. ${ }^{44-46}$ Moreover, rtMRI guidance does not necessitate the use of
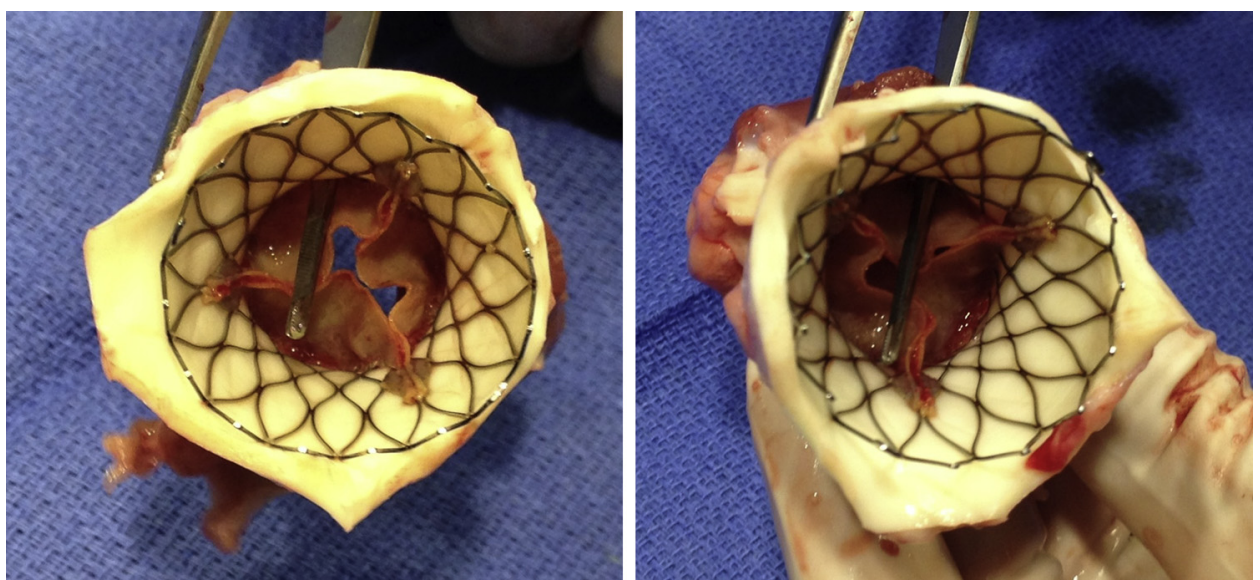

FIGURE 7. Necropsy results confirming the open coronary ostia. 
contrast media for a TAVR; however, fluoroscopy usually requires the use of contrast media to aid visualization. ${ }^{20-22}$ Contrast medium has been associated with multiple complications, including contrast-induced nephropathy and anaphylaxis. ${ }^{47}$ Although gadolinium can be used with the first-pass perfusion scan during postdeployment scanning to evaluate myocardial perfusion, it is not required to complete the procedure, especially if the patient has risk factors for contrast-induced nephropathy. ${ }^{40}$ rtMRI guidance may allow the use of TAVR in patients with renal insufficiency, which is currently a contraindication to the procedure. ${ }^{14,48}$

Although rtMRI has multiple benefits over conventional imaging modalities for TAVR, it also has several hurdles that need to be overcome before a clinical model can be proposed. These limitations include non-MRIcompatible implants and the high cost of a hybrid MRI setup. Unlike in fluoroscopy and CT, in MRI the continuous interaction between the magnetic field and ferromagnetic materials can lead to movement and the heating of metallic objects, with susbsequent damage to electronic circuits. These phenomena necessitate special attention to screening patients before rtMRI procedures. Imaging of patients with non-MRI-compatible implants, such as some implanted cardiac devices, is contraindicated. Before rtMRI-quided TAVR, patients would need to follow routine MRI screening protocols, including a standardized questionnaire to ensure that there are no contraindications to imaging. ${ }^{49-51}$ Patients with nonMRI-compatible implants will be excluded from rtMRI guidance.

Another hurdle is the technology and resources required to establish an rtMRI TAVR program, which can be a costly and time-consuming endeavor. Our setup includes a hybrid MRI catheterization suite. We have an MRI-compatible table in a procedure portion of the room, where the introducer sheath can be placed sterilely. The table is then remotely moved into the adjacent MRI bore for imaging and placement under rtMRI guidance. In the event of a surgical emergency, the MRI table can be retracted from the magnet and the patient immediately rolled into an adjacent fully equipped operating room. Both the MRI catheterization suite and operating room have full angiographic capabilities. Although we used general anesthesia for our animal experiments, the MRI catheterization suite has the appropriate equipment for performing the procedure under sedation or general anesthesia. The components include MRI-compatible anesthetic equipment as well as MRI-compatible telemetry, infusion pumps, and invasive hemodynamic monitoring. The operating room, adjacent to the MRI catheterization suite, has the capability for cardiopulmonary bypass. This setup is designed for a clinical model in which intraprocedural complications may dictate aborting the procedure for immediate cardiopulmonary bypass and operative repair.

\section{CONCLUSIONS}

Our results demonstrate that the Medtronic CoreValve system can be easily and effectively deployed with a left subclavian approach using rtMRI, an active guidewire, and a modified valve delivery catheter system in swine. rtMRI guidance is unique in that this single imaging modality can be successfully used for predeployment imaging, imaging guidance for valve deployment, and postdeployment scanning. Predeployment imaging allowed for precise and efficient measurement of the aortic annulus and aortic root to confirm their size. The TAVR procedure with rtMRI guidance allowed for adequate visualization of anatomic structures as well as the active guidewire, delivery device, and stent. Postdeployment imaging allowed immediate evaluation of valve function, as well as assessment for regurgitation and myocardial perfusion. Although rtMRI guidance has several limitations, including the cost and technical challenges of establishing a hybrid MRI suite with the necessary imaging equipment and trained medical staff, we believe that the benefits of this imaging modality far outweigh its drawbacks. We have been able to successfully demonstrate in several preclinical experiments the feasibility of rtMRI guidance in performing TAVR. We also have demonstrated the benefits of a single imaging modality for predeployment imaging, imaging guidance for valve deployment, and postdeployment scanning. This method eliminates the morbidity of radiation exposure, rapid ventricular pacing, contrast media renal toxicity, and a more invasive procedure. Although we do not envision that rtMRI will supplant the current modalities for TAVR, we do envision a significant role for this modality in the near future. The success of this preclinical model with the use of a commercially available TAVR bioprosthetic valve brings the rtMRI-guided approach closer to clinical reality.

\section{Conflict of Interest Statement}

Authors have nothing to disclose with regard to commercial support.

We thank Medtronic for generously providing the CoreValve bioprosthetic valves for these experiments. The authors had full freedom of investigation.

\footnotetext{
References

1. Coffey S, Cox B, Williams MJ. The prevalence, incidence, progression, and risks of aortic valve sclerosis: a systematic review and meta-analysis. J Am Coll Cardiol. 2014;63:2852-61.

2. Maganti K, Rigolin VH, Sarano ME, Bonow RO. Valvular heart disease: diagnosis and management. Mayo Clin Proc. 2010;85:483-500.

3. Stewart BF, Siscovick D, Lind BK, Gardin JM, Gottdiener JS, Smith VE, et al. Clinical factors associated with calcific aortic valve disease. Cardiovascular Health Study. J Am Coll Cardiol. 1997;29:630-4.

4. Nkomo VT, Gardin JM, Skelton TN, Gottdiener JS, Scott CG, EnriquezSarano M. Burden of valvular heart diseases: a population-based study. Lancet. 2006;368:1005-11.

5. Ross J Jr, Braunwald E. Aortic stenosis. Circulation. 1968;38(1 Suppl):61-7. 6. Carabello BA. Introduction to aortic stenosis. Circ Res. 2013;113:179-85.
} 
7. Turina J, Hess OM, Krayenbühl HP. Spontaneous course of aortic valve disease and indications for aortic valve replacement. Schweiz Med Wochensch. 1988; 118:508-16 (in German).

8. Leon MB, Smith CR, Mack M, Miller DC, Moses JW, Svensson LG, et al. Transcatheter aortic-valve implantation for aortic stenosis in patients who cannot undergo surgery. $N$ Engl J Med. 2010;363:1597-607.

9. Otto CM. Timing of aortic valve surgery. Heart. 2000;84:211-8.

10. Kelly TA, Rothbart RM, Cooper CM, Kaiser DL, Smucker ML, Gibson RS. Comparison of outcome of asymptomatic to symptomatic patients older than 20 years of age with valvular aortic stenosis. Am J Cardiol. 1988;61:123-30.

11. Saeedi M, Thomas A, Shellock FG. Evaluation of MRI issues at 3-Tesla for a transcatheter aortic valve replacement (TAVR) bioprosthesis. Magn Reson Imaging. 2015;33:497-501.

12. Kindzelski BA, Zhou Y, Horvath KA. Transmyocardial revascularization devices: technology update. Med Devices (Auckl). 2015;8:11-9.

13. Kindzelski BA, Li M, Mazilu D, Hunt T, Horvath KA. Real-time magnetic resonance-guided aortic valve replacement using Engager valve. Ann Thorac Surg. 2014;98:2194-9.

14. Horvath KA, Mazilu D, Cai J, Kindzelski B, Li M. Transapical sutureless aortic valve implantation under magnetic resonance imaging guidance: acute and short-term results. J Thorac Cardiovasc Surg. 2015;149:1067-72.

15. Hahn RT. Use of imaging for procedural guidance during transcatheter aortic valve replacement. Curr Opin Cardiol. 2013;28:512-7.

16. Bloomfield GS, Gillam LD, Hahn RT, Kapadia S, Leipsic J, Lerakis S, et al. A practical guide to multimodality imaging of transcatheter aortic valve replacement. JACC Cardiovasc Imaging. 2012;5:441-55.

17. Jilaihawi H, Kashif M, Fontana G, Furugen A, Shiota T, Friede G, et al. Cross-sectional computed tomographic assessment improves accuracy of aortic annular sizing for transcatheter aortic valve replacement and reduces the incidence of paravalvular aortic regurgitation. JAm Coll Cardiol. 2012;59:1275-86.

18. Husser O, Holzamer A, Resch M, Endemann DH, Nunez J, Bodi V, et al. Prosthesis sizing for transcatheter aortic valve implantation: comparison of three-dimensional transesophageal echocardiography with multislice computed tomography. Int J Cardiol. 2013;168:3431-8.

19. Smith LA, Dworakowski R, Bhan A, Delithanasis I, Hancock J, Maccarthy PA, et al. Real-time three-dimensional transesophageal echocardiography adds value to transcatheter aortic valve implantation. J Am Soc Echocardiogr. 2013;26:359-69.

20. McCullough PA, Wolyn R, Rocher LL, Levin RN, O'Neill WW. Acute renal failure after coronary intervention: incidence, risk factors, and relationship to mortality. Am J Med. 1997;103:368-75.

21. Bruschi G, De Marco F, Martinelli L, Klugmann S. CoreValve transcatheter self-expandable aortic bioprosthesis. Expert Rev Med Devices. 2013;10:15-26.

22. Horvath KA, Mazilu D, Guttman M, Zetts A, Hunt T, Li M. Midterm results of transapical aortic valve replacement via real-time magnetic resonance imaging guidance. J Thorac Cardiovasc Surg. 2010;139:424-30.

23. Saeed M, Hetts SW, English J, Wilson M. MR fluoroscopy in vascular and cardiac interventions (Review). Int J Cardiovasc Imaging. 2012;28:117-37.

24. Roberts TP, Hassenzahl WV, Hetts SW, Arenson RL. Remote control of catheter tip deflection: an opportunity for interventional MRI. Magn Reson Med. 2002;48: 1091-5.

25. Horvath KA, Mazilu D, Kocaturk O, Li M. Transapical aortic valve replacement under real-time magnetic resonance imaging guidance: experimental results with balloon-expandable and self-expanding stents. Eur J Cardiothorac Surg. 2011; 39:822-8.

26. CoreValve System (package insert). Minneapolis (MN): Medtronic, Inc; 2014.

27. Grube E, Laborde JC, Gerckens U, Felderhoff T, Sauren B, Buellesfeld L, et al. Percutaneous implantation of the CoreValve self-expanding valve prosthesis in high-risk patients with aortic valve disease: the Siegburg first-in-man study. Circulation. 2006;114:1616-24.

28. Kocaturk O, Kim AH, Saikus CE, Guttman MA, Faranesh AZ, Ozturk C, et al. Active two-channel $0.035^{\prime}$ guidewire for interventional cardiovascular MRI. $J$ Magn Reson Imaging. 2009:30:461-5.

29. Guttman MA, Kellman P, Dick AJ, Lederman RJ, McVeigh ER. Real-time accelerated interactive MRI with adaptive TSENSE and UNFOLD. Magn Reson Med. 2003;50:315-21.

30. McVeigh ER, Guttman MA, Lederman RJ, Li M, Kocaturk O, Hunt T, et al. Real-time interactive MRI-guided cardiac surgery: aortic valve replacement using a direct apical approach. Magn Reson Med. 2006;56:958-64.

31. Horvath KA, Guttman M, Li M, Lederman RJ, Mazilu D, Kocaturk O, et al. Beating heart aortic valve replacement using real-time MRI guidance. Innovations (Phila). 2007:2:51-5.
32. Horvath KA, Li M, Mazilu D, Guttman MA, McVeigh ER. Real-time magnetic resonance imaging guidance for cardiovascular procedures. Semin Thorac Cardiovasc Surg. 2007; 19:330-5.

33. Lederman RJ. Cardiovascular interventional magnetic resonance imaging. Circulation. 2005;112:3009-17.

34. Kellman P, Derbyshire JA, Agyeman KO, McVeigh ER, Arai AE. Extended coverage first-pass perfusion imaging using slice-interleaved TSENSE. Magn Reson Med. 2004:51:200-4.

35. Shanewise JS, Cheung AT, Aronson S, Stewart WJ, Weiss RL, Mark JB, et al ASE/SCA guidelines for performing a comprehensive intraoperative multiplane transesophageal echocardiography examination: recommendations of the American Society of Echocardiography Council for Intraoperative Echocardiography and the Society of Cardiovascular Anesthesiologists Task Force for Certification in Perioperative Transesophageal Echocardiography. Anesth Analg. 1999;89:870-84.

36. Moss RR, Ivens E, Pasupati S, Humphries K, Thompson CR, Munt B, et al. Role of echocardiography in percutaneous aortic valve implantation. JACC Cardiovasc Imaging. 2008;1:15-24.

37. Kahlert P, Parohl N, Albert J, Schäfer L, Reinhardt R, Kaiser GM, et al Towards real-time cardiovascular magnetic resonance-guided transarterial CoreValve implantation: in vivo evaluation in swine. J Cardiovasc Magn Reson. 2012;14:21.

38. Kahlert P, Eggebrecht H, Plicht B, Kraff O, McDougall I, Decker B, et al Towards real-time cardiovascular magnetic resonance-guided transarterial aortic valve implantation: in vitro evaluation and modification of existing devices. $J$ Cardiovasc Magn Reson. 2010;12:58.

39. Dick AJ, Guttman MA, Raman VK, Peters DC, Pessanha BS, Hill JM et al. Magnetic resonance fluoroscopy allows targeted delivery of mesenchymal stem cells to infarct borders in swine. Circulation. 2003;108 2899-904.

40. Quail MA, Nordmeyer J, Schievano S, Reinthaler M, Mullen MJ, Taylor AM Use of cardiovascular magnetic resonance imaging for TAVR assessment in patients with bioprosthetic aortic valves: comparison with computed tomography. Eur J Radiol. 2012;81:3912-7.

41. Tsang W, Bateman MG, Weinert L, Pellegrini G, Mor-Avi V, Sugeng L, et al. Accuracy of aortic annular measurements obtained from three-dimensional echocardiography, CT and MRI: human in vitro and in vivo studies. Heart 2012;98:1146-52.

42. Koos R, Altiok E, Mahnken AH, Neizel M, Dohmen G, Marx N, et al. Evaluation of aortic root for definition of prosthesis size by magnetic resonance imaging and cardiac computed tomography: implications for transcatheter aortic valve implantation. Int J Cardiol. 2012;158:353-8.

43. Tsekos NV, Khanicheh A, Christoforou E, Mavroidis C. Magnetic resonance-compatible robotic and mechatronics systems for image-guided interventions and rehabilitation: a review study. Annu Rev Biomed Eng. 2007; 9:351-87.

44. Grube E, Naber C, Abizaid A, Sousa E, Mendiz O, Lemos P, et al. Feasibility of transcatheter aortic valve implantation without balloon pre-dilation: a pilot study JACC Cardiovasc Interv. 2011;4:751-7.

45. Mendiz OA, Fraguas H, Lev GA, Valdivieso LR, Favaloro RR. Transcatheter aortic valve implantation without balloon predilation: a single-center pilot experience. Catheter Cardiovasc Interv. 2013;82:292-7.

46. Fiorina C, Maffeo D, Curello S, Lipartiti F, Chizzola G, D'Aloia A, et al. Direc transcatheter aortic valve implantation with self-expandable bioprosthesis: feasibility and safety. Cardiovasc Revasc Med. 2014;15:200-3.

47. Hong SJ, Wong JT, Bloch KJ. Reactions to radiocontrast media. Allergy Asthme Proc. 2002;23:347-51.

48. Al-Lamee R, Godino C, Colombo A. Transcatheter aortic valve implantation: current principles of patient and technique selection and future perspectives Circ Cardiovasc Interv. 2011;4:387-95.

49. Kanal E, Borgstede JP, Barkovich AJ, Bell C, Bradley WG, Etheridge S, et al. American College of Radiology white paper on MR safety: 2004 update and revisions. AJR Am J Roentgenol. 2004;182:1111-4.

50. Pohost GM. Editor's page: is CMR safe? J Cardiovasc Magn Reson. 2001;3:ix.

51. Sierra M, Machado C. Magnetic resonance imaging in patients with implantable cardiac devices. Rev Cardiovasc Med. 2008;9:232-8.

Key Words: transcatheter aortic valve replacement, realtime magnetic resonance image, swine animal model, Medtronic CoreValve 